\title{
Évaluation de la performance de cinq tests de diagnostic rapide pour le dépistage du virus de l'Immunodéficience Humaine (VIH 1 \& 2) au Tchad
}

\author{
Mbanga DJIMADOUM ${ }^{1,2,3}$, Nadlaou BESSIMBAYE ${ }^{1,3 *}$, Ali Mahamat MOUSSA ${ }^{3,4}$, \\ Abdelsalam TIDJANI ${ }^{3}$, Mbaidoum NARASSEM ${ }^{5}$, Maxime Djimadengar NARBÉ $^{1}$, \\ Alexis SANGBA ${ }^{1}$ et Nicolas BARRO ${ }^{6}$
}

\footnotetext{
${ }^{1}$ Service des Laboratoires, Hôpital Général de Référence Nationale (HGRN) de N'Djamena, Tchad.

${ }^{2}$ Centre National de Transfusion Sanguine de N'Djamena BP 5524 N'Djamena, Tchad.

${ }^{3}$ Faculté des Sciences de la Santé Humaine (FSSH), Université de N'Djamena BP-1117 N'Djamena, Tchad, Tel: 0023522514444.

${ }^{4}$ Service de Médecine Interne et Gastroentérologie, Hôpital Générale de Référence Nationale de N'Djamena, Tchad.

${ }^{5}$ Service des Maladies Infectieuses, Hôpital Général de Référence National de N’Djamena, Tchad.

${ }^{6}$ Laboratoire de Biologie Moléculaire, d'Epidémiologie et de Surveillance des Bactéries et Virus

Transmissibles par les Aliments (LaBESTA)/Ecole Doctorale Sciences et Technologies, Université Ouaga 1 Joseph KI-ZERBO, 03 BP 7021 Ouagadougou 03, Burkina Faso.

*Auteur correspondant ; E-mail: nadlaobes@yahoo.fr ; BP 130 N’Djamena,Tél: 00235516168.
}

\section{RESUME}

Dans la perspective de remplacer Immunocomb II Bispot HIV1/2 dans l'algorithme national de dépistage du VIH au Tchad, cinq tests de diagnostics rapides (TDR) étaient sélectionnés et soumis systématiquement à un panel d'échantillons (positif et négatif pour le VIH). Il s'agit de : ABON HIV1/2 O, MP Diagnostics MULTISURE HIV, SD Bioline HIV1/2 3.0, Exacto Pro test HIV et Alere HIV Combo. L'objectif principal de cette étude était de retenir un test rapide efficace et discriminatoire entre le VIH1 et VIH2 comme second test de l'algorithme national, et secondairement revérifier les performances des tests rapides déjà en usage dans notre contexte. Les cinq tests de détection Immunochromatographique étaient réalisés au laboratoire de l'Hôpital Général de Référence Nationale de N'Djamena en respectant les instructions des fabricants. L'étude a porté sur 100 sérums testés négatifs au VIH et 100 sérums des patients positifs au VIH1. Les données de l'évaluation ont été calculées en utilisant le tableau de contingence $2 \times 2$ des variables qualitatives nominales. Tous les tests évalués avaient une spécificité de 100\%, leur sensibilité a varié de 99 à 100\%, leur VPP à $100 \%$ et leur VPN de 99 à 100\%. Le MULTISURE HIV et SD Bioline HIV1/2 3.0 ont une sensibilité de $99 \%$ et une spécificité de 100\%. Cependant, des trois tests discriminatoires évalués, seul SD Bioline HIV1/2 3.0, a confirmé 99\% de VIH1. Cette étude, a permis d'évaluer les performances des TDR anciens et nouvellement mis sur le marché au Tchad avec des sérums recueillis localement.

(C) 2018 International Formulae Group. All rights reserved.

Mots clés: Evaluation de performance, diagnostic du VIH, discrimination, tests rapides, Tchad. 


\title{
Performance evaluation of five Rapid Diagnostic Tests for Human Immunodeficiency Virus (HIV) Screening in Chad.
}

\begin{abstract}
To replace Immunocomb II Bispot HIV1/2 in the national algorithm of screening of the HIV in the Chad, five tests of fast diagnoses ( TDR) were systematically selected and subjected to a panel of samples (positive and negative for the HIV). It relates to ABON HIV1/2 O, MP Diagnoses MULTISURE HIV, SD Bioline HIV1/2 3.0, test Exacto Pro HIV and Alere HIV combo. The main objective of this study was to retain an effective and discriminatory fast test enter the VIH1 and VIH2 as second test of the national algorithm, and secondarily verify the performances of the already used fast tests in our context. Five tests of detection Immunochromatographique realized in the laboratory of the General Hospital of National Reference of N'Djamena by respecting the instructions of the manufacturers. The study concerned 100 negative tested serums in the HIV and 100 serums of the positive patients in the VIH1. The data of the evaluation calculated by using the board of contingency $2 \times 2$ of the nominal qualitative variables. All the estimated tests had a $100 \%$ specificity, their sensibility varied 99 to $100 \%$, their 100\% VPP and their VPN of 99 to $100 \%$. The MULTISURE HIV and SD Bioline HIV1 / 23.0 has a 99\% sensibility and a 100\% specificity. However, three estimated discriminatory tests, only SD Bioline HIV1 / 2 3.0, confirmed 99\% of VIH1. This study allowed to estimate the performances of the former TDR and recently launched on the market in the Chad with meditative serums locally.
\end{abstract}

(C) 2018 International Formulae Group. All rights reserved.

Keywords: Performance evaluation, rapid diagnostic test, HIV diagnostic, discrimination, Chad.

\section{INTRODUCTION}

L'infection et la transmission du virus de l'immunodéficience humaine (VIH) constituent une préoccupation primordiale et mondiale pour l'organisation des Nations Unies pour le VIH-SIDA (ONUSIDA). Le monde a inversé la courbe du VIH depuis le pic de 2005, avec 1,2 millions de décès imputable au SIDA (ONUSIDA, 2015).

$\mathrm{Au}$ Tchad, la prévalence nationale du VIH était passée de 3,3\% en 2005 à $1,6 \%$ en 2015 (INSEED, 2016). Le nombre de personne vivant avec le VIH était estimé en fin 2016 à 110 000. Les nouvelles infections étaient estimées à 3800 dont 2800 adultes et 1000 enfants. La couverture pour le dépistage volontaire VIH était de $42,1 \%$, et environ 44.207 personnes vivant avec les VIH étaient sous antirétroviral soit une couverture de $40,2 \%$ dont $14 \%$ chez les enfants. La survie des personnes vivant avec le VIH (PVVIH) 12 mois après le traitement est de 67,8\% en 2016 (CNLS, 2016). Compte tenu de l'ampleur de cette infection, son diagnostic correct est une priorité pour la sécurité transfusionnelle, la prise en charge des malades et la surveillance épidémiologique (Gueu Baba, 2011; CDC/WHO/APHL, 2001; KouassiM'Bengue, 2008). Ainsi, dans la perspective $\mathrm{du}$ retrait d'un test de Diagnostic de l'infection à VIH entrant dans l'algorithme national de dépistage à savoir Immunocomb II Bispot HIV1/2 (Orgenics) qui est un test discriminant le VIH1 et VIH2, cinq tests de diagnostics rapides (TDR) ont été sélectionnés et soumis systématiquement à un panel d'échantillons (positif et négatif), en vue essentiellement de retenir un TDR discriminatoire de substitution pour certains tests, et accessoirement vérifier les performances pour les autres. Ces cinq tests sont: ABON HIV1/2 O, MP Diagnostics MULTISURE HIV, SD Bioline HIV1/2 3.0, Exacto Pro test HIV et Alere HIV Combo. Il est donc indispensable de mettre à disposition des programmes de santé des outils de diagnostic fiables, rapides, simples d'utilisation et de moindre coût.

Les TDR nouvellement mis sur le marché dans une région géographique donnée devraient toujours être évalués, avec des sérums recueillis localement, afin de 
déterminer les valeurs de sensibilité et de spécificité en fonction du profil épidémiologique du terrain (IMEA, 2014). En effet, le Tchad est un pays sahéliens où les conditions climatiques sont rudes. Dans ces conditions, les performances d'un TDR de sérodiagnostic diffèrent sûrement de celles observées dans des laboratoires de référence (Ndjoyi-Mbiguino and Bélec, 2005 ; Ouassa et al., 200 ; Dangnra et al., 2002 ; Amadou et al., 2005). Cependant, certains facteurs imposent la nécessité de recourir aux TDR. Il s'agit de l'exigence en équipement complexe et en consommables souvent difficilement accessibles à certains laboratoires. Il s'agit également d'examens relativement longs, parlant des techniques conventionnelles. Enfin, leur réalisation nécessite un certain niveau de technicité et de savoir-faire, limitant ainsi leur réalisation aux laboratoires disposant du matériel et du personnel adéquats (OMS/CDC/APHL, 2003). Pour ces raisons, l'évaluation d'une stratégie alternative de dépistage doit toujours être effectuée afin de prendre en compte les multiples facteurs (variabilité des souches virales, séroprévalence locale, facteurs opérationnels.

Le panel de contrôle négatif a été choisi parmi les sérums testés négatifs au VIH1 lors de la deuxième enquête nationale du VIH de 2014 et gardés dans des conditions standard de conservation. Le statut sérologique avait été déterminé à l'aide de deux tests ELISA (ELISA1: Microelisa Système VIRONOSTIKA HIV Ag/Ab et ELISA2: Siemens ENZYGNOST ANTIMeasles-Virus/(gM) de quatrième génération et Western blot 2.2 en cas de discordance. Les DBS contrôle négatif et positif du CDC ont servi de témoins à la validation des manipulations. Le panel de contrôle positif était choisi parmi les sérums des patients positifs au VIH1 et sous antirétroviraux (ARV) depuis au moins un an, et prélevés pour le dosage de la charge virale dans le cadre du bilan de suivi régulier.

L'objectif de cette étude était principalement d'évaluer les performances de cinq tests de diagnostic rapide afin de retenir un test efficace et discriminatoire entre le
VIH1 et VIH2 en vue de remplacer le second test de l'algorithme national du diagnostic de l'infection par le VIH au Tchad, et secondairement revérifier les performances des TDR déjà en usage dans notre contexte. Les TDR devraient obéir aux conditions suivantes:

- Bonne sensibilité- diagnostic précoce (Fenêtre sérologique)

- Bonne Spécificité;

- D'exécution facile;

- Accessible (financière);

- Détection du VIH-1 et VIH-2 et discrimination entre ces 2 virus ;

- Disponibilité du test dans le pays ;

- La température de conservation doit être étendue jusqu'à $+30{ }^{\circ} \mathrm{C}$;

- Le kit du TDR doit contenir outre les tests en bandelettes, cartes ou cassettes, tous les accessoires nécessaires et une notice d'utilisation ;

- Le résultat du test doit rester suffisamment stable après la lecture.

\section{MATERIEL ET METHODES}

Cadre, période et type d'étude

L'évaluation a été réalisée à l'Unité de Recherche et de la Formation du Service des laboratoires de l'Hôpital Général de Référence Nationale de N'Djamena (HGRN) le 30 août 2017. Il s'agissait d'évaluer cinq TDR VIH à l'aide de panel de sérums humains sélectionnés de manière anonyme et non corrélé. Ces échantillons ont été sélectionnés uniquement sur leur statut vis-à-vis du VIH 1 tel que décrit ci-dessous.

\section{Matériel}

Matériel Biologique les sérums sanguins suivants ont été choisis pour l'évaluation :

- Sérums prélevés pour la charge virale chez les patients qui ont été étiquetés VIH1+ (selon l'Algorithme National de dépistage du VIH) avec les tests de Determine HIV1/2 et Immunocomb II Bispot HIV1/2, et qui sont éligibles (Taux de CD4 inférieur à $350 \quad \mu 1)$ au traitement antirétroviral (ARV) depuis au moins une année comme sérums de contrôle positif ; 
- Sérums de contrôle choisis parmi les sérums testés négatifs lors de la deuxième enquête nationale de séroprévalence du VIH de 2014 et gardés dans des conditions standard de la conservation (congélation à -25 oC). Rappelons que les sérums étaient obtenus des plasmas correspondants après centrifugation (10 minutes à $1500 \mathrm{t} / \mathrm{min}$ ).

- Matériel physico-chimique, le matériel physico-chimique est composé de réactifs et des consommables pour chaque kit du test :

- ABON HIV1/2 O;

- MP Diagnostics MULTISURE HIV;

- SD Bioline HIV1/2 3.0;

- Exacto Pro test HIV;

- Alere HIV Combo;

- DBS Positif control (ID\#: HPC-082114);

- DBS Négative control (ID\#: NC-081914).

L'évaluation de chaque TDR est faite en le testant systématiquement et en parallèle 100 échantillons du panel positifs aux VIH et 100 échantillons du panel négatifs au VIH soit 200 tests pour un TDR et 1000 tests effectués pour les cinq TDR.

Les cinq tests de détection Immunochromatographique utilisés détectent soit des antigènes du VIH soit des anticorps anti-VIH, et leur utilisation avait respecté les instructions de chaque fabricant.
Méthodes de calcul de la sensibilité (Se), la spécificité ( $\mathrm{Sp}$ ), la valeur prédictive positive (VPP) et la valeur prédictive négative (VPN) des TDR évalués

- Sensibilité (Se) capacité d'un test à identifier correctement les personnes infectées par le VIH ;

- Spécificité ( $\mathrm{Sp})$ capacité d'un test à identifier correctement les personnes non infectées par le VIH ;

- Valeur Prédictive Positive (VPP) probabilité qu'une personne ayant résultat positif soit réellement infectée par VIH ;

- Valeur Prédictive Négative (VPN) probabilité qu'une personne ayant un résultat négatif ne soit pas infectée par VIH.

Le calcul de Se, Sp, VPP et VPN était effectué avec les variables nominales suivantes : infecté VIH/Non infecté VIH pour les sérums sanguins et Positif/Négatif pour chaque TDR.

Les logiciels Excel 2013 et Word 2013 ont été utilisés pour la saisie et analyse des données. Les données de l'évaluation ont été calculées en utilisant le tableau de contingence $2 \times 2$ des variables qualitatives nominales (Tableau 1).

Tableau 1: tableau de contingente $2 \times 2$ pour le calcul de Se, Sp, VPP et VPN.

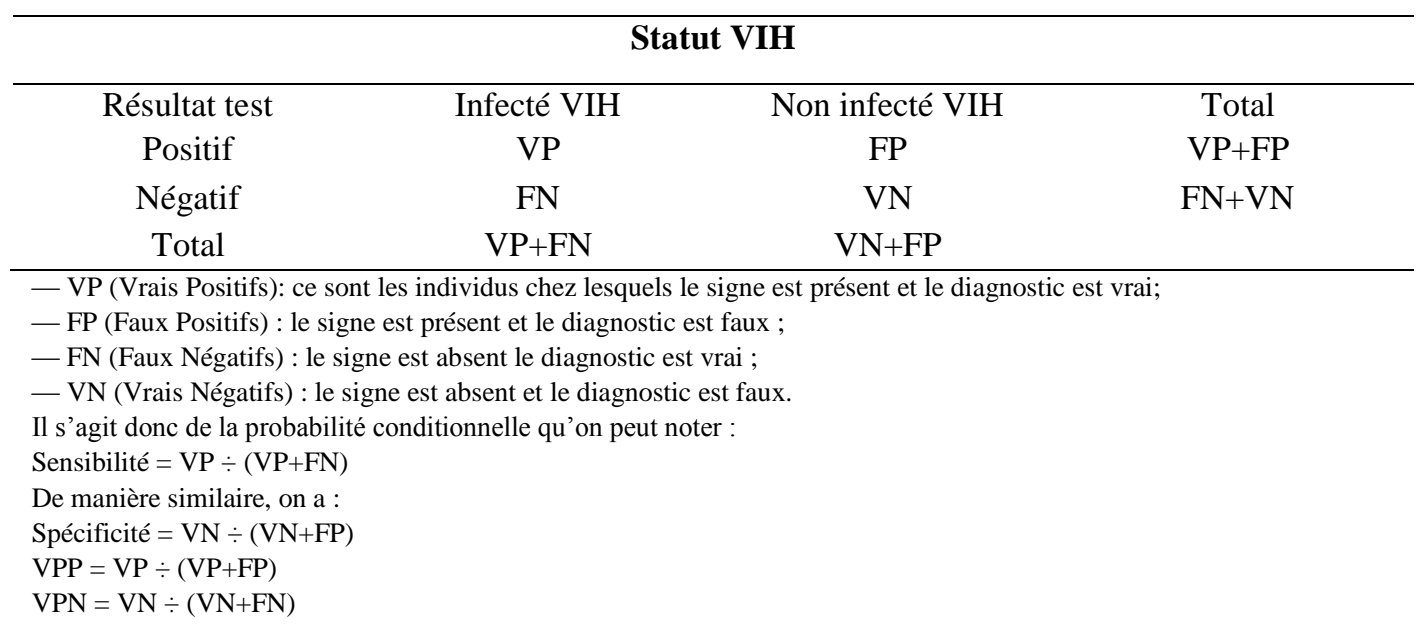




\section{RESULTATS}

\section{Détermination de la performance diagnostique des TDR}

L'évaluation des cinq tests de diagnostic rapide de l'infection par le VIH à l'aide d'un panel d'échantillons cliniques et ceux de la population a montré que tous peuvent être réalisés avec de sang total, plasma et sérum. La possibilité d'utiliser du sang total est un avantage important pour les centres de dépistage périphérique (pas de possibilité de centrifugation et ni de décantation du sérum). Les TDR étudiés se caractérisaient par une sensibilité allant de 99\% pour les moins sensibles (le MULTISURE HIV et le SD Bioline HIV1/2 3.0) à $100 \%$ pour $\mathrm{ABON}$ HIV1/2 $\mathrm{O}$, Exacto Pro test HIV et Alere HIV Combo avec le panel Tchadien de 100 sérums positifs au VIH1, provenant des patients vivant à N'Djamena venant à l'HGRN pour la charge virale. Par contre elle est de $100 \%$ avec le panel tchadien de 100 sérums négatifs d'individus tchadiens issus de la deuxième enquête national de VIH en 2014 (Tableau 4).

Des trois tests discriminatoires, le TDR (ABON HIV1/2 O) a une bonne sensibilité et une bonne spécificité de 100\% (Tableau 4), mais celui-ci présente un défaut de stabilité pour les éventuelles contrôles de qualité interne et ou même externe. De plus il a détecté un nombre anormalement élevé du VIH2 (07) contrairement au profil épidémiologique du VIH au Tchad.

Des 100 tests positifs, le MP Diagnostics MULTISURE HIV a confirmé 99 positifs et un test était négatif. Parmi les 99 tests positifs, le MULTISURE HIV a détecté 64 antigènes (gp41 + gp120 + p24 + ligne de contrôle colorée) pour le VIH soit une proportion de 65\%, 7 gp41 avec ligne de contrôle de qualité du test colorée pour le VIH1(7\%), 11 (gp41 + gp120 + ligne de contrôle colorée) pour le VIH1 (11\%), 12 (gp41 + p24+ ligne de contrôle colorée) pour le VIH1 (11\%), 2 (gp120 + p24+ ligne de contrôle de qualité du test colorée) pour le VIH1 (2\%) soit au total 96\% du VIH1. Le MP Diagnostics MULTISURE HIV a détecté 1 (gp36 + P24+ ligne de contrôle interne du test colorée) pour le VIH2 et 2 cas de co-infection VIH1/VIH2 $(\mathrm{gp} 41+\mathrm{gp} 120+\mathrm{gp} 36+\mathrm{p} 24+$ ligne de contrôle colorée interne du test colorée).

Les tests non discriminatoires : Exacto Pro test HIV, et Alere HIV Combo ont présenté une sensibilité et une spécificité de $100 \%$ (Tableau 4).

Les autres paramètres (température, durée, discrimination) qui peuvent influer sur le choix des tests au Tchad sont résumés dans le Tableau 2. Le coût des tests et la facilité d'approvisionnement sont d'autres paramètres à discuter avec le fournisseur.

\section{Choix du meilleur test discriminatoire (Tableau 4)}

Le test SD Bioline HIV1/2 3.0 présente une bonne sensibilité (99\%) et une très bonne spécificité $(100 \%)$ pour les échantillons cliniques testés positifs au VIH-1; une sensibilité et une spécificité de $100 \%$ pour les 100 sérums sanguins de contrôle négatif. Il a comme autres avantages : la facilité de lecture et la stabilité des résultats, la discrimination entre le VIH1 et le VIH2. Par contre, le MP Diagnostics MULTISURE HIV est un test de diagnostic de précision plus importante pour la recherche, mais il présente deux inconvénients majeurs: la multiplicité des lignes tests sur la cassette et la possibilité de réactions croisées avec les sérums sanguins humains contenant d'autres virus pouvant augmenter les risques d'erreurs et limiter la reproductibilité (MP Diagnostics MULTISUREHIV, 2016). Le TDR (ABON HIV1/2 O) a une bonne sensibilité et une bonne spécificité de 100\% (Tableau 4), mais celui-ci présente un défaut de stabilité pour les éventuelles contrôles de qualité interne et ou même externe, en plus de détecter en excès le VIH2 qui est quasi exceptionnel au Tchad. 
M. DJIMADOUM et al. / Int. J. Biol. Chem. Sci. 12(5): 2162-2171, 2018

Tableau 2: caractéristiques essentielles des cinq TDR selon la notice du fabricant.

\begin{tabular}{|c|c|c|c|c|c|c|c|c|}
\hline TDR & VIH-1 Ag & VIH-2 Ag & Ag groupe 0 & Principe & $\begin{array}{l}\text { Température de } \\
\text { stockage }\left({ }^{\circ} \mathrm{C}\right)\end{array}$ & $\begin{array}{l}\text { Durée } \\
\text { (min) }\end{array}$ & $\begin{array}{l}\text { Discrimination } \\
\text { VIH1 et VIH2 }\end{array}$ & Fabricant \\
\hline ABON HIV1/2 O & Non précisé & Non précisé & + & IC & $2-30$ & 10 & Oui & $\begin{array}{l}\text { Biopharma (Hangzou) } \\
\text { Co., Ltd) }\end{array}$ \\
\hline Alere HIV Combo. & $\mathrm{P} 24$ & $\mathrm{P} 24$ & + & IC & $2-30$ & $20 / 20-40$ & Non & $\begin{array}{l}\text { Alere Medical Co., } \\
\text { Ltd }\end{array}$ \\
\hline Exacto Pro test HIV & Non précisé & Non précisé & + & IC & $2-30$ & 10 & Non & Biosinex \\
\hline MULTISURE HIV & Gp120, gp41, p24 & Gp36, p24 & + et $\mathrm{M}$ & IC & $2-28$ & 20 & $\begin{array}{lr}\text { Oui } & (97,55 \% \\
\text { VIH1 et } \quad 93,94 \\
\text { VIH2) }\end{array}$ & $\begin{array}{l}\text { MP Biomedicals Asia } \\
\text { Pacific Pte. Ltd }\end{array}$ \\
\hline SD Bioline HIV $1 / 2$ & $\mathrm{Gp} 41, \mathrm{p} 24$ & Gp36, p24 & + & IC & $1-30$ & $10-20$ & Oui & Standard \\
\hline 3.0 & & & & & & & & Diagnostics, inc \\
\hline
\end{tabular}


Tableau 3: valeurs relatives de Se, Sp, VPP et VPN des cinq TDR selon la notice de chaque fabricant (ABON HIV1/2 O, 2014 ; MP DiagnosticsMULTISUREHIV, 2016 ; Exacto, 2015 ; SD Bioline HIV1/2 3.0, 2016 ; Alere HIV Combo, 2016).

\begin{tabular}{|c|c|c|c|c|c|c|}
\hline \multirow[t]{2}{*}{ TDR } & \multicolumn{2}{|c|}{ Nombre d'échantillons appariés } & \multirow{2}{*}{$\begin{array}{l}\text { Se (\%) } \\
\text { (95\% IC) }\end{array}$} & \multirow{2}{*}{$\begin{array}{l}\mathrm{Sp}(\%) \\
\text { (95\% IC) }\end{array}$} & \multirow{2}{*}{$\begin{array}{l}\text { VPP (\%) } \\
(95 \% \text { IC) }\end{array}$} & \multirow{2}{*}{$\begin{array}{l}\text { VPN (\%) } \\
(\mathbf{9 5 \%} \text { IC) }\end{array}$} \\
\hline & positif & négatif & & & & \\
\hline ABON HIV $1 / 2 \mathrm{O}$ & $26 / 26$ & $50 / 50$ & $\begin{array}{l}99,9 \\
(97,7-100)\end{array}$ & $\begin{array}{l}99,8 \\
(99,6-99,9)\end{array}$ & Non précisé & Non précisé \\
\hline Alere HIV Combo. & $\begin{array}{l}\text { Ligne Ag-Ab } \\
422 / 422\end{array}$ & $\begin{array}{l}\text { Ligne Ag-Ab } \\
1747 / 1749\end{array}$ & $\begin{array}{l}\text { Ligne Ag-Ab } \\
(100)\end{array}$ & $\begin{array}{l}\text { Ligne Ag-Ab } \\
(99,72)\end{array}$ & Non précisé & Non précisé \\
\hline Exacto Pro test HIV & $410 / 410$ & 209/211 & $\begin{array}{l}99,9 \\
(99,47-100,0)\end{array}$ & $\begin{array}{l}98,5 \\
(98,98-99,78)\end{array}$ & Non précisé & Non précisé \\
\hline MULTISURE HIV & $801 / 801$ & $2039 / 2057$ & $\begin{array}{l}100,00 \\
(99,54- \\
100,00)\end{array}$ & $\begin{array}{l}99,12 \\
(98,62-99,48)\end{array}$ & $\begin{array}{l}97,80 \\
(96,55-98,69)\end{array}$ & $\begin{array}{l}100,00 \\
(99,82- \\
100,00)\end{array}$ \\
\hline SD Bioline HIV1/2 3.0 & $187 / 187$ & $511 / 512$ & $100(98-100)$ & $99,8(98,9-100)$ & Non précisé & Non précisé \\
\hline
\end{tabular}

Tableau 4: valeurs de Se, P, VPP, VPN des cinq TDR obtenues après l'évaluation.

\begin{tabular}{|c|c|c|c|c|c|c|c|c|c|}
\hline \multirow[t]{2}{*}{ TDR } & \multicolumn{2}{|c|}{ Nombre de sérums } & \multicolumn{2}{|c|}{$\begin{array}{l}\text { Paramètres } \\
\text { évaluation }\end{array}$} & calculés & après & \multicolumn{2}{|c|}{ Discrimination } & $\begin{array}{l}\text { Co- } \\
\text { infection }\end{array}$ \\
\hline & positif & négatif & $\begin{array}{l}\mathrm{Se} \\
(\%)\end{array}$ & $\begin{array}{l}\text { Sp } \\
(\%)\end{array}$ & $\begin{array}{l}\text { VPP } \\
(\%)\end{array}$ & VPN (\%) & $\begin{array}{l}\text { VIH-1 } \\
(\%)\end{array}$ & $\begin{array}{l}\text { VIH-2 } \\
(\%)\end{array}$ & $\begin{array}{ll}\text { VIH1 } & \text { et } \\
\text { VIH2 } & \end{array}$ \\
\hline ABON HIV1/2 O & $100 / 100$ & $100 / 100$ & 100 & 100 & 100 & 100 & 93 & 00,00 & 7,00 \\
\hline Alere HIV Combo. & $100 / 100$ & $100 / 100$ & 100 & 100 & 100 & 100 & Non & Non & Non \\
\hline Exacto Pro test HIV & $100 / 100$ & $100 / 100$ & 100 & 100 & 100 & 100 & Non & Non & Non \\
\hline MULTISURE HIV & $99 / 100$ & $100 / 100$ & 99 & 100 & 100 & 99 & 96 & 1,00 & 2,00 \\
\hline SD Bioline HIV1/2 3.0 & $99 / 100$ & $100 / 100$ & 99 & 100 & 100 & 99 & 99 & 00,00 & 00,00 \\
\hline
\end{tabular}

\section{DISCUSSION}

$\mathrm{Au}$ terme de ce travail qui portait sur l'évaluation de la performance des cinq TDR, tous les tests évalués ont présenté une sensibilité et une spécificité supérieure à $95 \%$ avec les sérums testés. Pour ce qui est des tests discriminatoires, le TDR (ABON HIV1/2 $\mathrm{O}$ a une bonne sensibilité et une bonne spécificité de $100 \%$ (Tableau 4), selon le type d'échantillon sanguin testé. Ces valeurs étaient les mêmes avec le sérum sanguin selon la notice du fabricant mais différentes des valeurs relatives obtenues après le test avec le sang entier, le plasma et le sérum sanguin (Tableau 3). Le test ABON HIV $1 / 2$ O présente un défaut de stabilité, car après les 10 minutes réglementaires pour la lecture des résultats, les lignes de contrôle interne du test et les lignes des tests VIH-1 et VIH-2 deviennent de plus en plus fine. De plus, lorsque les trois lignes apparaissent, il faut considérer l'une des lignes de test la plus 
colorée pour donner le résultat (ABON HIV1/2 O, 2014). De ce fait, il pourra être source de divergence d'interprétations selon les différents niveaux de la pyramide de notre système de santé. Le défaut de stabilité d'un test rapide due au temps de lecture et d'autres paramètres (température...), ont été également observés lors de l'évaluation de neuf trousses de dépistage de l'infection à VIH au Gabon (Ndjoyi-Mbiguino, et Bélec, 2005). En Côte d'Ivoire, sur six tests rapides évalués, quatre (4) tests répondaient aux critères de l'organisation mondiale de la santé à savoir une sensibilité supérieure à $99 \%$ et une spécificité supérieure à $95 \%$ mais deux (2) autres ont présenté des spécificités faibles avec le panel de sérums sanguins testés localement (Ouassa et al., 2007).

Les TDR (MP Diagnostics MULTISURE et HIV SD Bioline HIV1/2 3.0) présentent une sensibilité de $99 \%$ et une spécificité de $100 \%$ avec les sérums sanguins de contrôle positif testés (Tableau 4). Des valeurs inverses ont été observées par le fabricant (Tableau 3). Par contre le MULTISURE HIV et le SD Bioline HIV1/2 3.0 ont donné une réaction à $100 \%$ négative avec les sérums sanguins de contrôle négatif. Le test SD Bioline HIV1/2 3.0 présente une sensibilité de $99 \%$ et une spécificité de $100 \%$ pour les échantillons cliniques testés positifs au VIH-1 ; une sensibilité et une spécificité de $100 \%$ pour les 100 sérums sanguins de contrôle négatif comme le MP Diagnostics MULTISURE HIV, mais il semble plus spécifique car il confirme 99 cas de VIH-1 sur 100 sérums VIH-1+ (Tableau 4). Par ailleurs, une étude similaire a été réalisée au Panama en Amérique Latine pour mesurer les Performances du test de diagnostic rapide qui montré une sensibilité de $98,8 \%$ et une spécificité de 100\%, comparable à notre proportion pour ce qui est de SD Bioline HIV1/2 3.0 (Sandra et al., 2016). Par contre, le MP Diagnostics MULTISURE HIV n'a confirmé que 96 cas de VIH1 sur 100 sérums sanguins pour le contrôle positif VIH-1+ (Tableau 4). La spécificité de MP Diagnostics MULTISURE HIV pour le VIH-1 et VIH-2 a été observée de la même manière par le fabricant avec la confirmation du VIH-1 à 97,55\% et du VIH-2 à 93,94\% (Tableau 2). Le MP Diagnostics MULTISURE HIV est un test de diagnostic de précision plus importante pour la recherche, mais il présente deux inconvénients majeurs: la multiplicité des lignes tests sur la cassette et la possibilité de réactions croisées avec les sérums sanguins humains contenant d'autres virus pouvant augmenter les risques d'erreurs et limiter la reproductibilité (MP Diagnostics MULTISUREHIV, 2016). Par ailleurs, la multiplicité des étapes et des réactifs augmentent les risques d'erreurs et limitent le nombre de tests réalisables en séries ont été rapportés par Amadou et al. (2004) au Niger lors d'une évaluation de cinq tests rapides et de deux algorithmes pour le diagnostic de l'infection par le VIH.

Le de kit SD Bioline HIV1/2 3.0 contient des réactifs et consommables nécessaires pour la réalisation du test dans les centres de dépistage en périphérique au Tchad. Il a des qualités qui sont importantes à prendre en considération dans les conditions opérationnelles du Tchad : facilité d'exécution (mono-étape et discriminant), transport et stockage à température ambiante (Tableau 2). D'autre part, le SD Bioline HIV1/2 3.0 est disponible partout dans les formations sanitaires et les Centres de Dépistage Volontaire (CDV) au Tchad. L'identification d'un test rapide et discriminatoire pour un algorithme de dépistage du VIH en tenant compte de différents niveaux sanitaires et des conditions environnementales a été également rapportée par Sangaré (2003) au Mali. Le SD Bioline HIV1/2 3.0 est le plus robuste des cinq tests évalués. Il peut être proposé comme un test de criblage et de discrimination dans l'algorithme national au Tchad en remplacement d'Immunocomb II Bispot HIV1/2 (Orgenics).

Par contre les tests d'Exacto Pro test HIV, et Alere HIV Combo bien que n'étant pas discriminatoires entre le VIH-1 et VIH-2, ont cependant une très grande sensibilité et spécificité (Tableau 4), et peuvent être recommandé en cas d'algorithmes alternatifs. 


\section{Conclusion}

La présente étude a mis évidence une très bonne sensibilité (99\%) et spécificité $(100 \%)$ du Kit SD Bioline HIV1/2 3.0. Ce TDR s'est révélé être l'un des meilleurs outils pour la détection de VIH-1 et VIH-2 sur le terrain, que l'infection soit passée ou en cours d'évolution. Son introduction dans l'algorithme national pour le diagnostic de l'infection par le VIH au Tchad pourra être envisagée, tant sa réalisation est possible partout sur l'ensemble du territoire national, sa conservation à la température ambiante (1$30{ }^{\circ} \mathrm{C}$ ) et la possibilité de travailler sur un sang total et la rapidité du rendu de résultat (15 min) avec une stabilité suffisante. Mais en raison de la variabilité génétique du VIH, ce type d'évaluation devrait être régulièrement réalisé sur un panel de sérums sanguins humains récents et sur tous les nouveaux tests comme ceux déjà évalués.

\section{CONFLITS D'INTERETS}

Les auteurs déclarent n'avoir aucun conflit d'intérêts.

\section{CONTRIBUTIONS DES AUTEURS}

MD : Initiateur et concepteur du projet de la recherche; Rédaction préliminaire de l'article ; Révision critique et apport décisif au contenu intellectuel ; Approbation finale de la version à publier; Assume l'imputabilité de tous les aspects de la recherche notamment l'exactitude et à l'intégrité de toute partie de l'article. NB : Contributions substantielles à la conception du projet de la recherche et la rédaction des méthodes de la recherche; Contrôle et suivi des réalisations des tests au laboratoire; Acquisition, analyse statistique et l'interprétation des données; Rédaction préliminaire de l'article; Révision critique et apport décisif au contenu intellectuel ; Approbation finale de la version à publier; Assume l'imputabilité de tous les aspects de la recherche notamment l'exactitude et à l'intégrité de toute partie de l'article. AMM: Révision critique et apport décisif au contenu intellectuel ; Approbation finale de la version à publier. AT: Approbation finale de la version à publier. $\mathrm{MN}$ : Approbation finale de la version à publier. MDN : Contributions substantielles à la collecte des échantillons et la supervision de l'exécution des tests au laboratoire; Assume l'imputabilité pour tous les aspects de l'article. AS: Contributions substantielles à la collecte des échantillons et la supervision de l'exécution des tests au laboratoire; Assume l'imputabilité pour tous les aspects de l'article. NB: Contributions substantielles à la conception du projet de la recherche; Révision critique et apport décisif au contenu intellectuel; Approbation finale de la version à publier; Assume l'imputabilité de tous les aspects de la recherche notamment l'exactitude et à l'intégrité de toute partie de l'article.

\section{REMERCIEMENTS}

Nous exprimons notre profonde gratitude à l'égard du Ministère de la Santé Publique qui a encouragé cette étude. Nos remerciements vont également à l'endroit des représentants des maisons de fabrication des réactifs pour avoir fourni les réactifs nécessaires pour cette étude.

\section{REFERENCES}

ABON HIV1/2 O. 2014. Immunodeficiency Virus Rapid Test Device HIV1/2/O (catalogue: IHI-T402W), no 115610902 , IFU version 02. Human. Instruction for Use: Hangzhou, China ; 1-2.

Alere HIV Combo. 2016. Test rapide Immunochromatographique pour la détection de l'antigène $\mathrm{P} 24$ du VIH1 libre et des anticorps anti-VIH1 et antiVIH2 (REF 7D2842, 7D2843). Alere HIV Combo (juin 2016241336 R4). Notice, Chiba, Japan. www.alere.com/www.alerehiv.com; 110.

Amadou A, Kouka N, Elhadj Mahamane A, Chanteau S. 2005. Évaluation de cinq tests rapides et de deux algorithmes pour le diagnostic de l'infection par le VIH au Niger. Bull. Soc. Pathol. Exot., 98 (1) : 5-8.

CDC/WHO/APHL. 2001. Directives pour l'évaluation des techniques de dépistage du VIH en Afrique, élaborée lors d'une réunion de travail du 28 novembre au $1^{\mathrm{er}}$ décembre 2001 à Harare, au Zimbabwe.Rapport d'activité, P.72.

CNLS. 2016. Rapport d'activité/PSN 20142017, P.24.

Dagnra A Y, Prince David M, Gaba J, OuroAkpo, Segbena A Y, Ali-Edje K, Ehlan A, Bougoudogo F. 2002. Evaluation de la performance de huit tests de 
diagnostic de l'infection à VIH à Lomé (Togo). Méd. Trop., 62(5) : 501-510.

Exacto. 2015. Test rapide Immunochromatographique pour la détection des anticorps VIH (REF 857218 Version 01 FR). Exacto TEST HIV (Novembre 2015). Notice, Strasbourg Cedex, France; 1-4.

Gueu Baba R. 2011. Concordance entre les tests VIH à la bandelette et les tests rapides IMMUNOCOMBS II et GENIE II au Mali Université de Bamako. Thèse de Doctorat en Pharmacie, Bamako, P.99.

INSEED (Institut National de la Statistique, des Études Économiques et Démographiques) 2016. Enquête Démographique et de Santé et à Indicateurs Multiples au Tchad (EDSMICS). Rapport 2014-2015. IFC-DHS, P.655.

Kouassi-M'Bengue A, Boni-Cissé C, FayeKetté H, Kacou-N douba A, Sevede D, Méité S, Adou L, N drin G, Dosso M. 2008. Evaluation de 12 tests rapides de dépistage de l'infection à VIH à Abidjan de 1999-2006. Revue Bio-Africa., 6: 2124.

MP Diagnostics MULTISUREHIV. 2016. Test rapide Immunochromatographique pour la détection des anticorps VIH1 et VIH2 (REF : 43030-02). TEST RAPIDE MULTISURE VIH (Décembre 2016). Notice, Singapou, Asia, Bureau régional Eschwege, Allemagne ; 1-4.

IMEA (Institut de Médecine et d'Epidémiologie Appliquée) /FILM (Fondation Internationale Léon Mba). 2014. Rapport d'activité, P.68.
Ndjoyi-Mbiguino A, Bélec L. 2005. Évaluation de trousses de dépistage de l'infection à VIH au Gabon. Cahiers. Santé., 15(1) : 1-5.

ONUSIDA. 2015. Rapport d'activité 2015 sur la riposte au SIDA dans le monde. Rapport d'activité, 1-236. www.unaids.org.

Ouassa T, Inwoley A, Loukou Yao G, Coulibaly Erd, Combe P. 2007. Evaluation de six tests rapides pour le dépistage des anticorps anti-VIH1 et VIH2. J. Sci. Pharm. Biol., 8(2): 57-64. SD Bioline HIV1/2 3.0. 2016. Test rapide Immunochromatographique pour la détection des anticorps (IgG, IgM, IgA) du VIH1 et VIH2 REF 03FK10-04Fr-3/03FK16-04-Fr-3). SD Bioline HIV1/2 3.0 (août 2016). Notice, Boragahal-ro, Giheung-gu, Gyeonggi-do, Korea; 1-10.

OMS/CDC/APHL. 2003. Directives pour l'évaluation des techniques de dépistage $\mathrm{du} \mathrm{VIH}$ en Afrique. Document technique, p.71.

Sandra I, Juarez A, Aurelio E, Nuñez B, Margginna M, Aranda B, Dalis Mojica C, Andrea A, Kim D, Bharat Parekh D. 2016. Field Evaluation of Four Rapid Tests for Diagnosis of HIV Infection in Panama. J. Clin. Microbiol., 53(11): 3501-3506. DOI: 10.1128/JCM.0143215.

Sangaré DB. 2003. Identification d'un algorithme de dépistage du VIH par des tests rapides utilisables dans les centres de conseils et de dépistage volontaire (CCDV) au Mali Université de Bamako. Thèse de Doctorat en Pharmacie, Bamako, P.99. 